\title{
LARGE SIEVE INEQUALITY WITH CHARACTERS FOR POWERFUL MODULI
}

\author{
STEPHAN BAIER AND LIANGYI ZHAO
}

\begin{abstract}
In this paper we aim to generalize the results in 1, 2, 19 and develop a general formula for large sieve with characters to powerful moduli that will be an improvement to the result in [19].
\end{abstract}

keywords: large sieve inequality; power moduli.

Mathematics Subject Classification 2000: 11N35, 11L07, 11B57

\section{INTRODUCTION}

Throughout this paper, we reserve the symbols $c_{i}(i=1,2, \ldots)$ for absolute positive constants. Large sieve was an idea originated by J. V. Linnik [10] in 1941 while studying the distribution of quadratic non-residues. Refinements of this idea were made by many. In this paper, we develop a large sieve inequality for powerful moduli. More in particular, we aim to have an estimate for the following sum

$$
\sum_{q \leq Q} \sum_{\substack{a=1 \\(a, q)=1}}^{q^{k}}\left|\sum_{n=M+1}^{M+N} a_{n} e\left(\frac{a}{q^{k}} n\right)\right|^{2},
$$

where $k \geq 2$ is a natural number. In the sequel, let

$$
Z:=\sum_{n=M+1}^{M+N}\left|a_{n}\right|^{2} .
$$

With $k=1$ in (1.1), it is

$$
\ll\left(Q^{2}+N\right) Z \text {. }
$$

This is in fact the consequence of a more general result first introduced by H. Davenport and H. Halberstam [7] in which the Farey fractions in the outer sums of (1.1) can be replaced by any set of well-spaced points. Applying the said more general result, (1.1) is bounded above by

$$
\ll\left(Q^{k+1}+Q N\right) Z \text {, and } \ll\left(Q^{2 k}+N\right) Z
$$

(see [19]). Literature abound on the subject of the classical large sieve. See [3], [6], [7, 8], 10], 12], 13] and [14. In [19] it was proved that the sum (1.1) can be estimated by

$$
\ll\left(Q^{k+1}+\left(N Q^{1-1 / \kappa}+N^{1-1 / \kappa} Q^{1+k / \kappa}\right) N^{\varepsilon}\right) Z,
$$

where $\kappa:=2^{k-1}$ and the implied constant depends on $\varepsilon$ and $k$. Furthermore, when appropriate, some of the constants $c_{i}$ 's and the implied constants in $\ll$ in the remainder of this paper will depend on $\varepsilon$ or both $\varepsilon$ and $k$. In [1] and 2] this bound was improved for $k=2$. Extending the elementary method in [1] to higher power moduli, we here establish the following bound for (1.1).

Date: November 20, 2018. 
Theorem 1: We have

$$
\sum_{q \leq Q} \sum_{\substack{a=1 \\(a, q)=1}}^{q^{k}}\left|\sum_{n=M+1}^{M+N} a_{n} e\left(\frac{a}{q^{k}} n\right)\right|^{2} \ll(\log \log 10 N Q)^{k+1}\left(Q^{k+1}+N+N^{1 / 2+\varepsilon} Q^{k}\right) Z .
$$

For $k \geq 3$ Theorem 1 improves the classical bounds (1.3) as well as Zhao's bound (1.4) in the range $N^{1 /(2 k)+\varepsilon} \ll$ $Q \ll N^{(\kappa-2) /(2(k-1) \kappa-2 k)-\varepsilon}$. In particular, for $k=3$ we obtain an improvement in the range $N^{1 / 6+\varepsilon} \ll Q \ll$ $N^{1 / 5-\varepsilon}$. We note that for a large $k$ the exponent $(\kappa-2) /(2(k-1) \kappa-2 k)$ is close to $1 /(2(k-1))$.

Extending the Fourier analytic methods in [2, [19, we establish another bound for cubic moduli which improves the bounds (1.3), (1.4) in the range $N^{7 / 25+\varepsilon} \ll Q \ll N^{1 / 3-\varepsilon}$.

Theorem 2: Suppose that $1 \leq Q \leq N^{1 / 2}$. Then we have

$$
\sum_{q \leq Q} \sum_{\substack{a=1 \\(a, q)=1}}^{q^{3}}\left|\sum_{n=M+1}^{M+N} a_{n} e\left(\frac{a}{q^{3}} n\right)\right|^{2} \ll \begin{cases}N^{\varepsilon}\left(Q^{4}+N^{9 / 10} Q^{6 / 5}\right) Z, & \text { if } N^{7 / 24} \leq Q \leq N^{1 / 2}, \\ N Q^{6 / 7+\varepsilon} Z, & \text { if } 1 \leq Q<N^{7 / 24} .\end{cases}
$$

Unfortunately, our Fourier analytic method does not yield any improvement if $k \geq 4$.

\section{Proof of Theorem 1}

Let $\mathcal{S}$ be the set of $k$-th powers of natural numbers. Let $Q_{0} \geq \sqrt{N}$. Set

$$
\mathcal{S}\left(Q_{0}\right)=\mathcal{S} \cap\left(Q_{0}, 2 Q_{0}\right] .
$$

We first note, by classical large sieve, setting $Q=\sqrt{N}$ in (1.2),

$$
\sum_{q \leq \sqrt{N}} \sum_{\substack{a=1 \\(a, q)=1}}^{q}\left|\sum_{n=M+1}^{M+N} a_{n} e\left(\frac{a}{q} n\right)\right|^{2} \leq 2 N Z
$$

Let

$$
\mathcal{S}_{t}\left(Q_{0}\right)=\left\{q \in \mathbb{N}: t q \in \mathcal{S}\left(Q_{0}\right)\right\}
$$

Let $t=p_{1}^{v_{1}} \cdots p_{n}^{v_{n}}$ be the prime decomposition of $t$. Furthermore, let

$$
u_{i}:=\left\lceil\frac{v_{i}}{k}\right\rceil,
$$

where for $x \in \mathbb{R},\lceil x\rceil=\min \{k \in \mathbb{Z}: k \geq x\}$ is the ceiling of $x$. Moreover, set

$$
f_{t}=p_{1}^{u_{1}} \cdots p_{n}^{u_{n}} \text {. }
$$

Therefore, for all $q_{0}^{k}=q \in \mathcal{S}, q$ is divisible by $t$ if and only if $q_{0}$ is divisible by $f_{t}$. Therefore, we have

$$
\mathcal{S}_{t}\left(Q_{0}\right)=\left\{q_{1}^{k} g_{t}: Q_{0}^{1 / k} / f_{t}<q_{1} \leq\left(2 Q_{0}\right)^{1 / k} / f_{t}\right\},
$$

where

$$
g_{t}:=\frac{f_{t}^{k}}{t}
$$

Moreover we note that

$$
\mathcal{S}_{t}\left(Q_{0}\right) \subset\left(Q_{0} / t, 2 Q_{0} / t\right]
$$

and that

$$
\left|\mathcal{S}_{t}\left(Q_{0}\right)\right| \leq \frac{\left(2 Q_{0}\right)^{1 / k}}{f_{t}}
$$

We set for $m \in \mathbb{N}, l \in \mathbb{Z}$ with $(m, l)=1$

$$
A_{t}(u, m, l)=\max _{Q_{0} / t \leq y \leq 2 Q_{0} / t}\left|\left\{q \in \mathcal{S}_{t}\left(Q_{0}\right) \cap(y, y+u]: q \equiv l \bmod m\right\}\right| .
$$


Let $\delta_{t}(m, l)$ be the number of solutions $x$ to the congruence

$$
x^{k} g_{t} \equiv l \bmod m \text {. }
$$

We now use Theorem 2 in [1] with $Q_{0} \geq \sqrt{N}$ :

Theorem 3: Assume that for all $t \in \mathbb{N}, m \in \mathbb{N}, l \in \mathbb{Z}, u \in \mathbb{R}$ with $t \leq \sqrt{N}, m \leq \sqrt{N} / t,(m, l)=1$, $m Q_{0} / \sqrt{N} \leq u \leq Q_{0} / t$ the conditions

$$
\begin{gathered}
A_{t}(u, m, l) \leq C\left(1+\frac{\left|\mathcal{S}_{t}\left(Q_{0}\right)\right| / m}{Q_{0} / t} \cdot u\right) \delta_{t}(m, l), \\
\sum_{\substack{l=1 \\
(m, l)=1}}^{m} \delta_{t}(m, l) \leq m, \\
\delta_{t}(m, l) \leq X
\end{gathered}
$$

hold for some suitable positive numbers $C$ and $X$. Then,

$$
\sum_{q \in \mathcal{S}\left(Q_{0}\right)} \sum_{\substack{a=1 \\(a, q)=1}}^{q}\left|\sum_{n=M+1}^{M+N} a_{n} e\left(\frac{a}{q} n\right)\right|^{2} \leq c_{0} C\left(\min \left\{Q_{0} X, N\right\}+Q_{0}\right)\left(\sqrt{N} \log \log 10 N+\max _{r \leq \sqrt{N}} \sum_{t \mid r}\left|\mathcal{S}_{t}\left(Q_{0}\right)\right|\right) Z .
$$

First, we have to check the validity of the conditions (2.4), (2.5) and (2.6). Conditions (2.4) and (2.5) are obviously satisfied with $C$ absolute. We further suppose that $\left(g_{t}, m\right)=1$ for otherwise $\delta_{t}(m, l)=0$ since $(m, l)=1$. Therefore, we must estimate the number of solutions to

$$
x^{k} \equiv \overline{g_{t}} l \bmod m \text {, }
$$

where $\overline{g_{t}}$ is the multiplicative inverse of $g_{t}$ modulo $m$. By the virtue of the Chinese remainder theorem, it suffices to estimate the number of solutions to (2.8) with $m$ as a prime power, say $m=p^{e}$, for $p \in \mathbb{P}$ and $e \in \mathbb{N}$. Note that the function

$$
\sigma_{k}:\left(\mathbb{Z} / p^{e} \mathbb{Z}\right)^{*} \longrightarrow\left(\mathbb{Z} / p^{e} \mathbb{Z}\right)^{*}: x \longrightarrow x^{k}
$$

is an endomorphism. Hence it is enough to estimate the size of its $\operatorname{kernel} \operatorname{ker}\left(\sigma_{k}\right)$. If $k=\pi_{1}^{a_{1}} \cdots \pi_{h}^{a_{h}}$ is the prime decomposition of $k$, then

$$
\sigma_{k}=\prod_{i=1}^{h} \sigma_{\pi_{i}}^{a_{i}}
$$

Thus,

$$
\left|\operatorname{ker} \sigma_{k}\right| \leq \prod_{i=1}^{h}\left|\operatorname{ker} \sigma_{\pi_{i}}\right|^{a_{i}}
$$

Hence, it suffices to estimate the size of $\mid$ ker $\sigma_{\pi} \mid$ for prime numbers $\pi$.

For $p \in \mathbb{P}$,

$$
x^{\pi}-1 \equiv 0 \bmod p
$$

has at most $\pi$ solutions. By elementary result (see [15], for example), a solution, $a$ mod $p^{e}$ with $e \geq 1$, of the congruence

$$
x^{\pi}-1 \equiv 0 \bmod p^{e}
$$

lifts to more than one solution to

$$
x^{\pi}-1 \equiv 0 \bmod p^{e+1}
$$

only when $p \mid \pi a^{\pi-1}$ and $p^{e+1} \mid a^{\pi}-1$. If $p \neq \pi, p \mid \pi a^{\pi-1}$ implies $p \mid a$, but it is not possible that $p^{e+1} \mid a^{\pi}-1$ as $\left(a^{\pi}-1, a\right)=1$. Thus, in this case (2.10) has at most $\pi$ solutions for all $e \geq 1$. In the following, we consider the case $p=\pi$. 
By Fermat's little theorem, there exists only one solution of the congruence

$$
x^{\pi}-1 \equiv 0 \bmod \pi,
$$

namely $1 \bmod \pi$. This solution lifts to exactly $\pi$ solutions to

$$
x^{\pi}-1 \equiv 0 \bmod \pi^{2},
$$

namely

$$
1,1+\pi, 1+2 \pi, \ldots, 1+(\pi-1) \pi \bmod \pi^{2} .
$$

More generally, if $a \bmod \pi^{e}$ is a solution to

$$
x^{\pi}-1 \equiv 0 \bmod \pi^{e},
$$

then, if $a$ lifts to solutions to

$$
x^{\pi}-1 \equiv 0 \bmod \pi^{e+1},
$$

they are of the form

$$
a, a+\pi^{e}, a+2 \pi^{e}, \ldots, a+(\pi-1) \pi^{e} \bmod \pi^{e+1} .
$$

Assume there are $j_{1}, j_{2} \in\{0, \ldots, \pi-1\}, j_{1} \neq j_{2}$ such that both $a+j_{1} \pi^{e}$ and $a+j_{2} \pi^{e}$ lift to solutions modulo $\pi^{e+2}$. Then $\pi^{e+2} \mid\left(a+j_{1} \pi^{e}\right)^{\pi}-1$ and $\pi^{e+2} \mid\left(a+j_{2} \pi^{e}\right)^{\pi}-1$, hence

$$
\left(a+j_{1} \pi^{e}\right)^{\pi}-\left(a+j_{2} \pi^{e}\right)^{\pi}=\left(j_{1}-j_{2}\right) \pi^{e} \sum_{i=0}^{\pi-1}\left(a+j_{1} \pi^{e}\right)^{\pi-1-i}\left(a+j_{2} \pi^{e}\right)^{i}
$$

is divisible by $\pi^{e+2}$. If $e \geq 2$, this implies $a \equiv 0 \bmod \pi$, but then $a$ cannot be a solution to (2.11). Therefore, if $e \geq 2$, only one of the solutions (2.12) lifts to a solution modulo $\pi^{e+2}$. From this we infer that the number of solutions to 2.11) never exceeds $\pi^{2}$, i.e.

Combining this with (2.9), we get

$$
\mid \text { ker } \sigma_{\pi} \mid \leq \pi^{2} .
$$

$$
\mid \text { ker } \sigma_{k} \mid \leq k^{2} \text {. }
$$

Therefore, by the Chinese remainder theorem, we obtain

$$
\delta_{t}(m, l) \leq k^{2 \omega(m)},
$$

where $\omega(m)$ is the number of distinct prime divisors of $m$. Since $2^{\omega(m)}$ is the number of square-free divisors of $m$, we have

$$
k^{2 \omega(m)} \leq \tau(m)^{2 \log _{2} k} \ll m^{\varepsilon},
$$

where $\tau(m)$ is the number of divisors of $m$. Thus, if $m \leq \sqrt{N}$, (2.6) holds with

$$
X \ll N^{\varepsilon} \text {. }
$$

Now, by Theorem 3 ,

$$
\sum_{q \in \mathcal{S}\left(Q_{0}\right)} \sum_{\substack{a=1 \\(a, q)=1}}^{q}\left|\sum_{n=M+1}^{M+N} a_{n} e\left(\frac{a}{q} n\right)\right|^{2}
$$

is majorized by

$$
\ll\left(\min \left\{Q_{0} N^{\varepsilon}, N\right\}+Q_{0}\right)\left(\sqrt{N} \log \log (10 N)+\max _{r \leq \sqrt{N}} \sum_{t \mid r} Q_{0}^{1 / k} f_{t}^{-1}\right) Z .
$$

The function

$$
G(r)=\sum_{t \mid r} \frac{1}{f_{t}}
$$

is clearly multiplicative. If $r$ is a prime power $p^{v}$, then

$$
G(r) \leq 1+k\left(\frac{1}{p}+\frac{1}{p^{2}}+\ldots\right)=1+\frac{k}{p-1} \leq\left(1+\frac{1}{p-1}\right)^{k}=\left(\frac{p^{v}}{\varphi\left(p^{v}\right)}\right)^{k} .
$$


Hence, for all $r \in \mathbb{N}$ we have

$$
G(r) \leq\left(\frac{r}{\varphi(r)}\right)^{k} \ll(\log \log 10 r)^{k}
$$

Hence (2.13) is

$$
\ll\left(\log \log 10 N Q_{0}\right)^{k+1}\left(\sqrt{N}+Q_{0}^{1 / k}\right)\left(\min \left\{Q_{0} N^{\varepsilon}, N\right\}+Q_{0}\right) .
$$

The above is always majorized by

$$
\ll\left(\log \log 10 N Q_{0}\right)^{k+1}\left(Q_{0}^{1+1 / k}+N^{1 / 2+\varepsilon} Q_{0}\right) .
$$

Summing over all relevant dyadic intervals and combining with (2.1), we see that (1.1) is majorized by

$$
\ll(\log \log 10 N Q)^{k+1}\left(Q^{k+1}+N+N^{1 / 2+\varepsilon} Q^{k}\right) Z .
$$

Therefore, our result follows.

\section{Proof of Theorem 2}

3.1. Reduction to Farey fractions in short intervals. As in [1, [2], our starting point is the following general large sieve inequality.

Lemma 1: Let $\left(\alpha_{r}\right)_{r \in \mathbb{N}}$ be a sequence of real numbers. Suppose that $0<\Delta \leq 1 / 2$ and $R \in \mathbb{N}$. Put

$$
K(\Delta):=\max _{\alpha \in \mathbb{R}} \sum_{\substack{r=1 \\\left\|\alpha_{r}-\alpha\right\| \leq \Delta}}^{R} 1,
$$

where $\|x\|$ denotes the distance of a real $x$ to its closest integer. Then

$$
\sum_{r=1}^{R}\left|S\left(\alpha_{r}\right)\right|^{2} \leq c_{1} K(\Delta)\left(N+\Delta^{-1}\right) Z
$$

In the sequel, we suppose that $\mathcal{S}$ is the set of cubes of natural numbers and that $\alpha_{1}, \ldots, \alpha_{R}$ is the sequence of Farey fractions $a / q$ with $q \in \mathcal{S}\left(Q_{0}\right), 1 \leq a \leq q$ and $(a, q)=1$, where $Q_{0} \geq 1$. We further suppose that $\alpha \in \mathbb{R}$ and $0<\Delta \leq 1 / 2$. Put

$$
I(\alpha):=[\alpha-\Delta, \alpha+\Delta] \text { and } P(\alpha):=\sum_{\substack{q \in \mathcal{S} \cap\left(Q_{0}, 2 Q_{0}\right] \\(a, q)=1 \\ a / q \in I(\alpha)}} 1 .
$$

Then we have

$$
K(\Delta)=\max _{\alpha \in \mathbb{R}} P(\alpha) .
$$

Therefore, the proof of Theorem 2 reduces to estimating $P(\alpha)$.

As in 11] and 2], we begin with an idea of D. Wolke [18. Let $\tau$ be a positive number with

$$
1 \leq \tau \leq \frac{1}{\sqrt{\Delta}}
$$

In [1] and 2] we put $\tau:=1 / \sqrt{\Delta}$, but in fact our method works for all $\tau$ satisfying (3.2). We will later fix $\tau$ in an optimal manner. In the said earlier papers, $\tau=1 / \sqrt{\Delta}$ was the optimal choice.

By Dirichlet's approximation theorem, $\alpha$ can be written in the form

$$
\alpha=\frac{b}{r}+z,
$$

where

$$
r \leq \tau,(b, r)=1,|z| \leq \frac{1}{r \tau}
$$

Thus, it suffices to estimate $P(b / r+z)$ for all $b, r, z$ satisfying (3.3). 
We further note that we can restrict ourselves to the case when

$$
z \geq \Delta .
$$

If $|z|<\Delta$, then

Furthermore, we have

$$
P(\alpha) \leq P\left(\frac{b}{r}-\Delta\right)+P\left(\frac{b}{r}+\Delta\right)
$$

$$
\Delta \leq \frac{1}{\tau^{2}} \leq \frac{1}{r \tau} .
$$

Therefore this case can be reduced to the case $|z|=\Delta$. Moreover, as $P(\alpha)=P(-\alpha)$, we can choose $z$ positive. So we can assume (3.4), without any loss of generality.

Summarizing the above observations, we deduce

Lemma 2: We have

$$
K(\Delta) \leq 2 \max _{\substack{r \in \mathbb{N} \\ r \leq \tau}} \max _{\substack{b \in \mathbb{Z} \\(b, r)=1}} \max _{\Delta \leq z \leq 1 /(\tau r)} P\left(\frac{b}{r}+z\right)
$$

3.2. Estimation of $P(b / r+z)$ - first way. We now prove a first estimate for $P(b / r+z)$ by using some results in 11. In the sequel, we suppose that the conditions (3.2), (3.3) and (3.4) are satisfied.

By inequality (41) in [1], we have

$$
P\left(\frac{b}{r}+z\right) \leq 1+6 \sum_{t \mid r} \sum_{\substack{0<m \leq 4 r z Q_{0} / t \\(m, r / t)=1}} A_{t}\left(\frac{\Delta Q_{0}}{t z}, \frac{r}{t},-\bar{b} m\right),
$$

where $A_{t}(u, m, l)$ is defined as in (2.3) and $\bar{b}$ is the multiplicative inverse of $b$ modulo $r$. By the results of section 2 , for $\mathcal{S}$ the set of cubes, the conditions (2.4), (2.5) and (2.6) with $X=\Delta^{-\varepsilon}$ are satisfied for all $t \in \mathbb{N}, m \in \mathbb{N}$, $l \in \mathbb{Z}, u \in \mathbb{R}$ with $t \leq \tau, m \leq \tau / t,(m, l)=1, m Q_{0} / \tau \leq u \leq Q_{0} / t$. Conditions (2.4) and (2.6) imply

$$
\sum_{\substack{0<m \leq 4 r z \\(m, r / t)=1}} A_{t}\left(\frac{\Delta Q_{0}}{t z}, \frac{r}{t},-\bar{b} m\right) \leq C\left(1+\frac{\Delta t\left|\mathcal{S}_{t}\left(Q_{0}\right)\right|}{r z}\right) \frac{4 r z Q_{0} X}{t}
$$

From (3.6), 3.7) and

$$
\sum_{t \mid r} \frac{1}{t} \leq \prod_{p \mid r}\left(1+\frac{1}{p}+\frac{1}{p^{2}}+\ldots\right)=\prod_{p \mid r} \frac{p}{p-1}=\frac{r}{\varphi(r)} \leq c_{2} \log \log 10 r
$$

we derive

$$
P\left(\frac{b}{r}+z\right) \leq 1+c_{3} Q_{0} X\left(r z \log \log 10 r+\Delta \sum_{t \mid r}\left|\mathcal{S}_{t}\left(Q_{0}\right)\right|\right) .
$$

Furthermore, by (2.2) and (2.14), we have

$$
\sum_{t \mid r}\left|\mathcal{S}\left(Q_{0}\right)\right| \ll(\log \log 10 r)^{3} Q_{0}^{1 / 3} .
$$

Thus, from (3.8) and the fact that $r \leq \tau=\Delta^{-1 / 2}$, we obtain

Proposition 1: Let $\mathcal{S}$ be the set of cubes of natural numbers. Suppose that the conditions [3.2), 3.3) and 3.4) are satisfied. Then we have

$$
P\left(\frac{b}{r}+z\right) \leq 1+c_{4} \Delta^{-\varepsilon}\left(Q_{0}^{4 / 3} \Delta+Q_{0} r z\right) .
$$


3.3. Estimation of $P(b / r+z)$ - second way. We now prove a second estimate for $P(b / r+z)$ by extending the Fourier analytic methods in [2, [19] to cubic moduli. The following bound for $P(b / r+z)$ can be proved in the same manner as Lemma 2 in [2].

Lemma 3: Let $\mathcal{S}$ be the set of cubes of natural numbers. Suppose that

$$
\frac{Q_{0} \Delta}{z} \leq \delta \leq Q_{0}
$$

Then,

$$
P\left(\frac{b}{r}+z\right) \leq c_{5}\left(1+\frac{1}{\delta} \int_{Q_{0}}^{2 Q_{0}} \Pi(\delta, y) \mathrm{d} y\right)
$$

where $I(\delta, y)=\left[y^{1 / 3}-c_{6} \delta / Q_{0}^{2 / 3}, y^{1 / 3}+c_{6} \delta / Q_{0}^{2 / 3}\right], J(\delta, y)=[(y-4 \delta) r z,(y+4 \delta) r z]$ and

$$
\Pi(\delta, y)=\sum_{q \in I(\delta, y)} \sum_{\substack{m \in J(\delta, y) \\ m \equiv-b q^{3} \\ m \neq 0}} 1 .
$$

We shall prove the following

Proposition 2: Let $\mathcal{S}$ be the set of cubes of natural numbers. Suppose that the conditions (3.2), (3.3) and 3.4) are satisfied. Then we have

$$
P\left(\frac{b}{r}+z\right) \leq c_{7} \Delta^{-\varepsilon}\left(Q_{0}^{4 / 3} \Delta+Q_{0}^{1 / 3} \Delta r^{-1 / 3} z^{-1}+\Delta^{-1 / 2}(r z)^{1 / 2}\right) .
$$

To derive Proposition 2 from Lemma 3, we need the following standard results from Fourier analysis.

Lemma 4: (Poisson summation formula, [5]) Let $f(x)$ be a complex-valued function on the real numbers that is piecewise continuous with only finitely many discontinuities and for all real numbers a satisfies

$$
f(a)=\frac{1}{2}\left(\lim _{x \rightarrow a^{-}} f(x)+\lim _{x \rightarrow a^{+}} f(x)\right) .
$$

Moreover, suppose that $f(x) \leq c_{8}(1+|x|)^{-c}$ for some $c>1$. Then,

$$
\sum_{n \in \mathbb{Z}} f(n)=\sum_{n \in \mathbb{Z}} \hat{f}(n), \text { where } \hat{f}(x):=\int_{-\infty}^{\infty} f(y) e(x y) \mathrm{d} y,
$$

the Fourier transform of $f(x)$.

Lemma 5: (see [19, for example) For $x \in \mathbb{R} \backslash\{0\}$ define

$$
\phi(x):=\left(\frac{\sin \pi x}{2 x}\right)^{2}, \text { and } \phi(0):=\lim _{x \rightarrow 0} \phi(x)=\frac{\pi^{2}}{4} .
$$

Then $\phi(x) \geq 1$ for $|x| \leq 1 / 2$, and the Fourier transform of the function $\phi(x)$ is

$$
\hat{\phi}(s)=\frac{\pi^{2}}{4} \max \{1-|s|, 0\} .
$$

Lemma 6: (see Lemma 3.1. in [9]) Let $F:[a, b] \rightarrow \mathbb{R}$ be twice differentiable. Assume that $\left|F^{\prime}(x)\right| \geq u>0$ for all $x \in[a, b]$. Then,

$$
\left|\int_{a}^{b} e^{i F(x)} \mathrm{d} x\right| \leq \frac{c_{9}}{u}
$$


Lemma 7: (see Lemma 4.3.1. in [4]) Let $F:[a, b] \rightarrow \mathbb{R}$ be twice continuously differentiable. Assume that $\left|F^{\prime \prime}(x)\right| \geq u>0$ for all $x \in[a, b]$. Then,

$$
\left|\int_{a}^{b} e^{i F(x)} \mathrm{d} x\right| \leq \frac{c_{10}}{\sqrt{u}}
$$

We shall also need the following estimates for cubic exponential sums.

Lemma 8: (see 11], 17]) Let $c \in \mathbb{N}, k, l \in \mathbb{Z}$ with $(k, c)=1$. Then,

$$
\sum_{d=1}^{c} e\left(\frac{k d^{3}+l d}{c}\right) \leq c_{11} c^{1 / 2+\varepsilon}(l, c) .
$$

Furthermore,

$$
\sum_{d=1}^{c} e\left(\frac{k d^{3}}{c}\right) \leq c_{11} c^{2 / 3}
$$

Proof of Proposition 2: We put

$$
\delta:=\frac{Q_{0} \Delta}{z}
$$

By Lemma 5, (3.12) can be estimated by

$$
\Pi(\delta, y) \leq \sum_{q \in \mathbb{Z}} \phi\left(\frac{q-y^{1 / 3}}{2 c_{6} \delta / Q_{0}^{2 / 3}}\right) \sum_{\substack{m \in \mathbb{Z} \\ m \equiv-b q^{3} \bmod r}} \phi\left(\frac{m-y r z}{8 \delta r z}\right) .
$$
into

Using Lemma 4 after a linear change of variables, we transform the inner sum on the right-hand side of (3.15)

$$
\sum_{\substack{m \in \mathbb{Z} \\ m \equiv-b q^{3} \bmod r}} \phi\left(\frac{m-y r z}{8 \delta r z}\right)=8 \delta z \sum_{j \in \mathbb{Z}} e\left(\frac{j b q^{3}}{r}+j y z\right) \hat{\phi}(8 j \delta z) .
$$

Therefore, we get for the double sum on the right-hand side of (3.15)

$$
\begin{aligned}
\sum_{q \in \mathbb{Z}} \phi\left(\frac{q-y^{1 / 3}}{2 c_{6} \delta / Q_{0}^{2 / 3}}\right) \sum_{\substack{m \in \mathbb{Z} \\
m \equiv-b q^{3} \bmod r}} \phi\left(\frac{m-y r z}{8 \delta r z}\right) \\
=8 \delta z \sum_{j \in \mathbb{Z}} e(j y z) \hat{\phi}(8 j \delta z) \sum_{d=1}^{\tilde{r}} e\left(\frac{\tilde{j} b d^{3}}{\tilde{r}}\right) \sum_{\substack{k \in \mathbb{Z} \\
k \equiv d}} \phi\left(\frac{k-y^{1 / 3}}{2 c_{6} \delta / Q_{0}^{2 / 3}}\right),
\end{aligned}
$$

where $\tilde{r}:=r /(r, j)$ and $\tilde{j}:=j /(r, j)$. Again using Lemma 4 after a linear change of variables, we transform the inner sum on the right-hand side of (3.16) into

$$
\sum_{\substack{k \in \mathbb{Z} \\ k \equiv d}} \phi\left(\frac{k-y^{1 / 3}}{2 c_{6} \delta / Q_{0}^{2 / 3}}\right)=\frac{2 c_{6} \delta}{\tilde{r} Q_{0}^{2 / 3}} \sum_{l \in \mathbb{Z}} e\left(l \cdot \frac{d-y^{1 / 3}}{\tilde{r}}\right) \hat{\phi}\left(\frac{2 c_{6} l \delta}{\tilde{r} Q_{0}^{2 / 3}}\right) .
$$


From (3.16) and (3.17), we obtain

$$
\begin{aligned}
& \frac{1}{\delta} \int_{Q_{0}}^{2 Q_{0}} \sum_{q \in \mathbb{Z}} \phi\left(\frac{q-y^{1 / 3}}{2 c_{6} \delta / Q_{0}^{2 / 3}}\right) \sum_{\substack{m \in \mathbb{Z} \\
m \equiv-b q^{3} \bmod r}} \phi\left(\frac{m-y r z}{8 \delta r z}\right) \mathrm{d} y \\
& \quad \leq \frac{16 c_{6} \delta z}{Q_{0}^{2 / 3}} \sum_{j \in \mathbb{Z}} \frac{\hat{\phi}(8 j \delta z)}{\tilde{r}} \sum_{l \in \mathbb{Z}} \hat{\phi}\left(\frac{2 c_{6} l \delta}{\tilde{r} Q_{0}^{2 / 3}}\right)\left|\sum_{d=1}^{\tilde{r}} e\left(\frac{\tilde{j} b d^{3}+l d}{\tilde{r}}\right) \int_{Q_{0}}^{2 Q_{0}} e\left(j y z-l \cdot \frac{y^{1 / 3}}{\tilde{r}}\right) \mathrm{d} y\right| .
\end{aligned}
$$

Applying the Lemmas 5 and 8 to the right-hand side of (3.18), and taking $r \leq 1 / \sqrt{\Delta}$ by (3.2) and (3.3) into account, we deduce

$$
\begin{aligned}
& \frac{1}{\delta} \int_{Q_{0}}^{2 Q_{0}} \sum_{q \in \mathbb{Z}} \phi\left(\frac{q-y^{1 / 3}}{c_{6} \delta / Q_{0}^{2 / 3}}\right) \sum_{\substack{m \in \mathbb{Z} \\
m \equiv-b q^{3} \bmod r}} \phi\left(\frac{m-y r z}{8 \delta r z}\right) \mathrm{d} y \\
& \quad \leq \frac{c_{12} \delta z \Delta^{-\varepsilon}}{Q_{0}^{2 / 3}}\left(\sum_{|j| \leq 1 /(8 \delta z)} \frac{1}{\sqrt{\tilde{r}}} \sum_{\substack{|l| \leq\left(\tilde{r} Q_{0}^{2 / 3}\right) /\left(2 c_{6} \delta\right) \\
l \neq 0}}(l, \tilde{r})\left|\int_{Q_{0}}^{2 Q_{0}} e\left(j y z-l \cdot \frac{y^{1 / 3}}{\tilde{r}}\right) \mathrm{d} y\right|+\sum_{|j| \leq 1 /(8 \delta z)} \frac{1}{\sqrt[3]{\tilde{r}}}\left|\int_{Q_{0}}^{2 Q_{0}} e(j y z) \mathrm{d} y\right| \mid\right.
\end{aligned}
$$

If $j \neq 0$, then

$$
\left|\int_{Q_{0}}^{2 Q_{0}} e(j y z) \mathrm{d} y\right| \leq \frac{1}{|j| z}
$$

If $j=0$ and $l \neq 0$, then

$$
\left|\int_{Q_{0}}^{2 Q_{0}} e\left(j y z-l \cdot \frac{y^{1 / 3}}{\tilde{r}}\right) \mathrm{d} y\right| \leq \frac{c_{13} Q_{0}^{2 / 3}}{|l|}
$$

by Lemma 6 (take into account that $\tilde{r}=1$ if $j=0$ ). If $j \neq 0$ and $l \neq 0$, then Lemma 7 yields

$$
\left|\int_{Q_{0}}^{2 Q_{0}} e\left(j y z-l \cdot \frac{y^{1 / 3}}{\tilde{r}}\right) \mathrm{d} y\right| \leq \frac{c_{14} \sqrt{\tilde{r}} Q_{0}^{5 / 6}}{\sqrt{|l|}}
$$

Therefore, the right-hand side of (3.19) is majorized by

$$
\leq c_{15} \delta \Delta^{-\varepsilon}\left(z Q_{0}^{1 / 3}+\frac{1}{Q_{0}^{2 / 3}} \sum_{1 \leq j \leq 1 /(8 \delta z)} \frac{1}{j \sqrt[3]{r}}+z \sum_{1 \leq l \leq Q_{0}^{2 / 3} /\left(2 c_{6} \delta\right)} \frac{1}{l}+z Q_{0}^{1 / 6} \sum_{1 \leq j \leq 1 /(8 \delta z)} \sum_{1 \leq l \leq \tilde{r} Q_{0}^{2 / 3} /\left(2 c_{6} \delta\right)} \frac{(l, \tilde{r})}{\sqrt{l}}\right) .
$$

Now, we estimate the sums in the last line of (3.20). Using (3.2), (3.3) and (3.14), we obtain

$$
\sum_{1 \leq l \leq Q_{0}^{2 / 3} /\left(2 c_{6} \delta\right)} \frac{1}{l} \leq c_{16} \Delta^{-\varepsilon}
$$

Using the definition of $\tilde{r},(3.2),(3.3)$ and (3.14), we obtain

$$
\sum_{1 \leq j \leq 1 /(8 \delta z)} \frac{1}{j \sqrt[3]{\tilde{r}}}=\frac{1}{\sqrt[3]{r}} \sum_{t \mid r} \sqrt[3]{t} \sum_{\substack{1 \leq j \leq 1 /(8 \delta z) \\(r, j)=t}} \frac{1}{j} \leq \frac{c_{17} \Delta^{-\varepsilon}}{\sqrt[3]{r}} \sum_{t \mid r} t^{-2 / 3} \leq c_{18} \Delta^{-2 \varepsilon} r^{-1 / 3} .
$$


For $A \geq 1$, we have

$$
\sum_{1 \leq l \leq A} \frac{(l, \tilde{r})}{\sqrt{l}} \leq \sum_{t \mid \tilde{r}} t \sum_{1 \leq l \leq A / t} \frac{1}{\sqrt{l t}} \ll \sqrt{A} \sum_{t \mid \tilde{r}} 1 \ll \tilde{r}^{\varepsilon} \sqrt{A}
$$

Therefore,

$$
\sum_{1 \leq j \leq 1 /(8 \delta z)} \sum_{1 \leq l \leq \tilde{r} Q_{0}^{2 / 3} /\left(2 c_{6} \delta\right)} \frac{(l, \tilde{r})}{\sqrt{l}} \leq \frac{c_{19} \Delta^{-\varepsilon} Q_{0}^{1 / 3}}{\sqrt{\delta}} \sum_{1 \leq j \leq 1 /(8 \delta z)} \sqrt{\tilde{r}}
$$

Using the definition of $\tilde{r}$, we obtain

$$
\sum_{1 \leq j \leq 1 /(8 \delta z)} \sqrt{\tilde{r}}=\sqrt{r} \sum_{t \mid r} \frac{1}{\sqrt{t}} \sum_{\substack{1 \leq j \leq 1 /(8 \delta z) \\(r, j)=t}} 1 \leq \frac{\sqrt{r}}{8 \delta z} \sum_{t \mid r} \frac{1}{t^{3 / 2}} \leq \frac{c_{20} \sqrt{r}}{\delta z} .
$$

Combining Lemma 3 and (3.193.24), we obtain

$$
P\left(\frac{b}{r}+z\right) \leq c_{7} \Delta^{-3 \varepsilon}\left(1+\delta z Q_{0}^{1 / 3}+\delta Q_{0}^{-2 / 3} r^{-1 / 3}+\delta^{-1 / 2} Q_{0}^{1 / 2} \sqrt{r}\right) .
$$

From (3.14) and (3.25), we infer the desired estimate. Note that the first term in the right-hand side of (3.25) can be absorbed into the last term on the right-hand side of (3.13) by (3.4).

3.4. Final proof of Theorem 2. Combining Propositions 1,2 and (3.3), we obtain

$$
P\left(\frac{b}{r}+z\right) \leq c_{21} \Delta^{-\varepsilon}\left(Q_{0}^{4 / 3} \Delta+\min \left\{Q_{0} r z, Q_{0}^{1 / 3} \Delta r^{-1 / 3} z^{-1}\right\}+\Delta^{-1 / 2} \tau^{-1 / 2}\right) .
$$

If

$$
z \leq \Delta^{1 / 2} Q_{0}^{-1 / 3} r^{-2 / 3}
$$

then

$$
\min \left\{Q_{0} r z, Q_{0}^{1 / 3} \Delta r^{-1 / 3} z^{-1}\right\}=Q_{0} r z \leq Q_{0}^{2 / 3} \Delta^{1 / 2} r^{1 / 3} .
$$

If

$$
z>\Delta^{1 / 2} Q_{0}^{-1 / 3} r^{-2 / 3}
$$

then

$$
\min \left\{Q_{0} r z, Q_{0}^{1 / 3} \Delta r^{-1 / 3} z^{-1}\right\}=Q_{0}^{1 / 3} \Delta r^{-1 / 3} z^{-1} \leq Q_{0}^{2 / 3} \Delta^{1 / 2} r^{1 / 3}
$$

From the above inequalities and (3.3), we deduce

$$
\min \left\{Q_{0} r z, Q_{0}^{1 / 3} \Delta r^{-1 / 2} z^{-1}\right\} \leq Q_{0}^{2 / 3} \Delta^{1 / 2} r^{1 / 3} \leq Q_{0}^{2 / 3} \Delta^{1 / 2} \tau^{1 / 3} .
$$

Combining (3.26) and (3.27), we get

$$
P\left(\frac{b}{r}+z\right) \leq c_{22} \Delta^{-\varepsilon}\left(Q_{0}^{4 / 3} \Delta \tau^{\varepsilon}+Q_{0}^{2 / 3} \Delta^{1 / 2} \tau^{1 / 3+\varepsilon}+\Delta^{-1 / 2} \tau^{-1 / 2}\right)
$$

Now we choose

$$
\tau:=\left\{\begin{array}{ll}
N^{6 / 5} Q_{0}^{-4 / 5}, & \text { if } N^{7 / 8} \leq Q_{0} \leq N^{3 / 2}, \\
Q_{0}^{4 / 7}, & \text { if } 1 \leq Q_{0}<N^{7 / 8},
\end{array} \text { and } \Delta:= \begin{cases}N^{-1}, & \text { if } N^{7 / 8} \leq Q_{0} \leq N^{3 / 2}, \\
Q_{0}^{-8 / 7}, & \text { if } 1 \leq Q_{0}<N^{7 / 8} .\end{cases}\right.
$$

Then the condition (3.2) is satisfied in each case, and from (3.28) and Lemmas 1,2, we obtain

$$
\sum_{Q_{0}^{1 / 3} \leq q \leq\left(2 Q_{0}\right)^{1 / 3}} \sum_{\substack{a=1 \\(a, q)=1}}^{q^{3}}\left|S\left(\frac{a}{q^{3}}\right)\right|^{2} \ll \begin{cases}N^{\varepsilon}\left(Q_{0}^{4 / 3}+N^{9 / 10} Q_{0}^{2 / 5}\right) Z, & \text { if } N^{7 / 8} \leq Q_{0} \leq N^{3 / 2}, \\ N Q_{0}^{2 / 7+\varepsilon} Z, & \text { if } 1 \leq Q_{0}<N^{7 / 8} .\end{cases}
$$

We can divide the interval $[1, Q]$ into $O(\log Q)$ subintervals of the form $\left[Q_{0}^{1 / 3},\left(2 Q_{0}\right)^{1 / 3}\right]$, where $1 \leq Q_{0} \leq Q^{3}$. Hence, the result of Theorem 2 follows from (3.29). 


\section{ACKNOWLEDGMENTS}

This paper was written when the first-named author held a postdoctoral position at the Harish-Chandra Research Institute at Allahabad (India) and the second-named author was supported by a postdoctoral fellowship at the University of Toronto. The authors wish to thank these institutions for their financial support.

\section{REFERENCES}

[1] S. Baier, On the large sieve with a sparse set of moduli, preprint.

[2] S. Baier, The large sieve with square moduli, preprint.

[3] E. Bombieri and H. Davenport, Some inequalities involving trigonometrical polynomials, Annali Scuola Normale Superiore Pisa 23 (1969) 223-241.

[4] J. Brüdern, Einführung in die analytische Zahlentheorie, Springer-Verlag, Berlin ect., 1995.

[5] D. Bump, Automorphic Forms and Representations, Cambridge Stud. Adv. Math. 55, Cambridge Univ. Press, Cambridge, 1996.

[6] H. Davenport, Multiplicative Number Theory, Third Edition, Graduate Texts in Mathematics, 74, Springer-Verlag, New York, etc., 2000.

[7] H. Davenport and H. Halberstam, The values of a trigonometric polynomial at well spaced points, Mathematika 13 (1966) 91-96, Corrigendum and addendum, Mathematika 14 (1967) 299-232.

[8] P. X. Gallagher, The large sieve, Mathematika 14 (1967) 14-20.

[9] S.W. Graham, G. Kolesnik, Van der Corput's Method of Exponential Sums, Cambridge University Press, Cambridge ect., 1991.

[10] J. V. Linnik, The large sieve, Doklady Akad. nauk SSSR 36 (1941) 119-120.

[11] L. K. Hua, On exponential sums, Sci. Record 1 (1957) 1-4.

[12] H. L. Montgomery, Topics in Multiplicative Number Theory, Lecture Notes in Mathematics, 227, Spring-Verlag, Berlin, etc., 1971.

[13] H. L. Montgomery, The analytic principles of large sieve, Bull. Amer. Math. Soc., 84 (1978) 547-567.

[14] H. L. Montgomery and R. C. Vaughan, The Large Sieve, Mathematika 20 (1973) $119-134$.

[15] I. Niven, H.S. Zuckerman, H.L. Montgomery, An introduction to the Theory of Numbers, John Wiley \& Sons, New York, 1991.

[16] R.C. Vaughan, The Hardy-Littlewood Method, Cambridge University Press, Cambridge, 1997.

[17] I.M. Vinogradov, The Method of Trigonometrical Sums in the Theory of Numbers, Trav. Inst. Math. Stekloff $23,1947$.

[18] D. Wolke, On the large sieve with primes, Acta Math. Acad. Sci. Hungar. 22 (1971/72) $239-247$.

[19] L. Zhao, Large sieve inequality with characters to square moduli, Acta Arith. 112 (2004) $297-308$.

Stephan Baier

Harish-Chandra Research Institute, Chhatnag Road, Jhusi, Allahabad 211 019, India

Email: sbaier@mri.ernet.in

Liangyi Zhao

Department of Mathematics, University of Toronto, 100 Saint George Street, Toronto, ON M5S 3G3, Canada

Email: lzhao@math.toronto.edu 